\title{
Childhood aggression, withdrawal and likeability, and the use of health care later: a longitudinal study
}

\author{
Caroline E. Temcheff PhD, Lisa A. Serbin PhD, Alexa Martin-Storey PhD, Dale M. Stack PhD, \\ Paul Hastings PhD, Jane Ledingham PhD, Alex E. Schwartzman PhD
}

See related commentary by Stewart-Brown on page 2083 and at www.cmaj.ca/lookup/doi/10.1503/cmaj.111754

\begin{abstract}
Background: Literature suggests that early patterns of aggressive behaviour in both girls and boys are predictive of a variety of health risks in adulthood. However, longitudinal examination of the predictive links between childhood aggression, negative physical health outcomes in adulthood and overall use of health care has not been done. We looked at use of health care and a variety of physical health outcomes in adulthood to extend the current body of knowledge regarding the long-term negative sequelae of childhood aggression.
\end{abstract}

Methods: Participants of the Concordia Longitudinal Risk Project were eligible for the current study if they had received medical care in the province of Quebec between 1992 and 2006, and if we were able to retrieve their medical and education records. Our primary outcome was use of the health care system, as determined using records from the Régie de l'assurance maladie du Québec and the Ministère de la santé et des services sociaux. Our controlled variables were socioeconomic status of the neighbourhood in which partici- pants lived in 1986 and level of education. We used hierarchical multiple regression to explore the association between childhood behaviour and physical health in adulthood.

Results: During the 15-year period studied, childhood agression corresponded to an increase in medical visits $(8.1 \%$ per 1 standard deviation increase in agression), and injuries $(10.7 \%)$ or lifestyle-related illnesses $(44.2 \%)$, visits to specialists $(6.2 \%)$ and visits to emergency departments (12.4\%). We saw a positive relation between social withdrawal during childhood and government-funded visits to dentists. Peer-rated likeability during childhood showed negative relations with use of health care (overall), medical visits due to injuries and government-funded visits to dentists.

Interpretation: Childhood aggression is a health risk that should be considered when designing interventions to improve public health and diminish the costs of medical services, particularly when considering interventions targeting children and families.
$\mathrm{P}$ redicting the use of health services among adults is gaining importance in Canada and elsewhere. As health care budgets decrease and the Canadian population ages, it is important to identify and understand the predictors of poor health in adulthood, as well as the protective factors that could lead to fewer medical interventions being needed.

A developing body of literature is beginning to show that, in addition to genetic and physiologic factors, personality and behaviour influence lifetime health trajectories and outcomes. There are several reasons to believe that aggressive behaviour, starting in childhood, may be a possible risk factor for a number of health problems and increased use of health services later in life. Childhood aggression has been shown to predict such health risks as not completing high school, ${ }^{1}$ teen pregnancy and single motherhood, ${ }^{2}$ poverty, ${ }^{3,4}$ engaging in high-risk and/or unprotected sex ${ }^{5,6}$ and dangerous driving. ${ }^{6,7}$

Government medical records and data on the use of health services drawn from a prospective longitudinal study provide a unique opportunity to examine the role of early childhood behaviour as it relates to health outcomes in adulthood. Research from the Concordia Longitudinal Risk Project using these types of data has shown that childhood aggression is predictive of overall use of health services, medical visits due to injuries and medical visits due to lifestyle-related illnesses. ${ }^{8-10}$ However, previous studies have only looked at a limited number of predictors of health and indicators of service use. Our goal was to
Competing interests: Caroline Temcheff and Alexa Martin-Storey received doctora fellowships from the Social Sciences and Humanities Research Council. Lisa Serbin and Dale Stack have received grants and support for travel from the Canadian Institutes of Health Research (CIHR); they have been reimbursed for expenses by Concordia University; and they will be receiving grants from the

Social Sciences and

Humanities Research

Council and Fonds de recherche société et culture. Paul Hastings has received grants from the CIHR, National Institute of Mental Health (NIMH) and the Fetzer Institute; he is a consultant for NIMH and the Association of State and Provincial Psychology Boards; and he receives royalties from Guilford Press. No other competing interests were declared.

This article has been peer reviewed.

Correspondence to:

Caroline E. Temcheff; caroline.temcheff @usherbrooke.ca.

CMAJ 2011. DOI:10.1503 /cmaj.091830 
extend previous research by including records on service use from a greater number of years in addition to a new predictor - the level of poverty of the neighbourhood in which participants spent their late adolescences and young adulthoods. This new predictor is an indicator of both socioeconomic status and the environmental influences that participants may have been exposed to during the early, formative years of their development as an adult. We included additional indicators of service use to better understand the relationship between childhood behaviour and subsequent use of health and medical services.

We expected childhood aggression to increase the use of health services. Previous data from the Concordia Longitudinal Risk Project ${ }^{9,10}$ and other studies ${ }^{11-13}$ show that childhood aggression in girls was predictive of poorer gynecologic health (particularly in late adolescence and early adulthood) and an increased number of early pregnancies. Although the focus of our study was on physical health and use of services associated with childhood aggression, the inclusion of childhood social withdrawal and childhood likeability are strengths of the Concordia Project. The longitudinal sequelae of these childhood behavioural and social characteristics are less well-documented and have rarely been studied longitudinally in the context of physical health. We expected childhood social withdrawal and likeability to lead to less overall use of health care and to be protective, specifically against injuries and obstetric and gynecologic conditions.

\section{Methods}

\section{Study population}

The methods of the Concordia Longitudinal Risk Project have been described in detail elsewhere. ${ }^{8}$ Briefly, from 1976 to 1978,4109 children in grades 1, 4 and 7 were screened for participation in the project. The goal of the project was to study the long-term sequelae of maladaptive behaviour in childhood. Participants lived in neighbourhoods with low socioeconomic status in Montréal, Quebec.

Children were asked to nominate up to four boys and four girls in their class who best matched each item on the Pupil Evaluation Inventory,${ }^{14}$ a peer-nomination instrument. The inventory consists of 34 items covering 3 factors: aggression, withdrawal and likeability. Scores on the inventory have been shown to be highly reliable. ${ }^{14,15}$ A more detailed description of the inventory is in Appendix 1 (available at www.cmaj.ca /lookup/suppl/doi:10.1503/cmaj.091830/-/DC1).

Participants of the Concordia Longitudinal Risk
Project were eligible for our study if they received medical care in the province of Quebec between 1992 and 2006. This period corresponds to the young to middle adulthoods of the participants (in 2006, participants had a mean age of $39.2 \mathrm{yr}$ [standard deviation (SD) 2.6, range 34-45]). Furthermore, participants were eligible for our study if we were able to retrieve information on the highest level of education they achieved. Of the original 4109 participants in the Concordia project, 3913 participated in our study (95.2\%).

All procedures were reviewed by the Concordia University institutional review board for conformity with ethical requirements for research. We used an encrypted identity-protecting coding procedure that was reviewed by the Commission d'accès à l'information du Québec before it permitted the release of Medicare records for research purposes. Analyses were performed on entirely denominalized data. It is worth noting that the ethical guidelines regarding parental consent have changed in the 30 years since the Concordia Project's inception; the "opt-out" approach to parental consent would no longer be considered sufficient in Canada.

\section{Sources of data}

Permission to retrieve information on health care use and admissions to hospital was granted by the Commission d'accès à l'information du Québec. We obtained data on the health services used by our 3913 participants from the Régie de l'assurance maladie du Québec (the insurance plan covering all permanent residents of the province, which pays for all medical services) and the Ministère de la santé et des services sociaux.

The medical records detailed all contacts with the health care system between 1992 and 2006, including information on medical procedures performed, assessments and diagnoses. All data were connected to a sequential number that differed from our own identification number to preserve the confidentiality of the records.

We used information from the Canada Census, 1986 , to calculate the level of poverty of the neighbourhoods in which participants lived in 1986.

We obtained the provincial education records of all participants from the Ministry of Education, Leisure and Sport to determine the highest level of education completed by each participant.

\section{Outcome variables}

We identified nine outcome variables: total number of medical visits (not including obstetric and gynecologic visits to allow for comparison between sexes), medical visits for injuries, medical visits for lifestyle-related illness (i.e., obesity, type 2 diabetes, ulcers, alcohol dependence, 
drug use); visits to medical specialists, visits to emergency departments, visits to dentists, admissions to hospital, medical visits for infections and gynecologic and obstetric visits (women only). A list of the medical codes included in each outcome can be seen in Appendix 2 (available at www.cmaj.ca/lookup/suppl/doi:10.1503 /cmaj.091830/-/DC1).

\section{Editor's note}

Table 1 may be unfamiliar to many of our readers. All of the variables in the table have been rescaled from the units used to measure them, such as the Pupil Evaluation Inventory score for aggression or the number of admissions to hospital. A consequence of this rescaling is that all of the values are now expressed in terms of how large they are in relation to the underlying distribution of values. An increase of one unit in this rescaled (standardized) score corresponds to an increase in that score that is the same as one standard deviation in the underlying distribution of scores. Thus, a change of one unit in the standardized score is quite large; for normally distributed data, a range of four standard deviations centered on the middle value would cover $95 \%$ of the data.

The associations in Table 1 represent the change in visits, expressed as proportions of a standard deviation, in response to a change of one standard deviation in the variables of interest (listed in the first column). Table 2 represents the same assocations, but with change in actual number of visits in response to a standardized change in the variables in the first column.

\section{Control variables}

We identified two control variables: socioeconomic status and level of education.

The socioeconomic status of the neighbourhoods in which our participants lived was measured in 1986, when the mean age of participants was about 19 years. Neighbourhoods were identified using the first three characters of the postal codes of participants' homes or schools. We determined the socioeconomic status of each neighbourhood using the proportion of people in the neighbourhood who had not completed high school, the proportion of single parents, the rate of unemployment and the proportion of families with annual incomes of less than \$20 000 .

\section{Box 1: Timing of data collection for this study}

- Childhood, 1976-1978

Peer-nomination instrument used to measure aggression, social withdrawal, and likeability in childhood

\section{- Adolescence and early adulthood, 1986}

Poverty level of participants' neighbourhoods was obtained using the following data from the Census of Canada, 1986:

(i) the proportion of people in the neighbourhood who had not completed high school

(ii) the proportion of single parents in the neighbourhood

(iii) the local rate of unemployment rate

(iv) the proportion of families with an annual income of less than $\$ 20000$

\section{- Adulthood, 2002-2003}

The level of education achieved by each participant was obtained from provincial education records

- Adulthood, 2006

Medical records were obtained through the Régie de l'assurance maladie du Québec.

Table 1: Standardized regression coefficients showing the relation between childhood characteristics and adult health outcomes

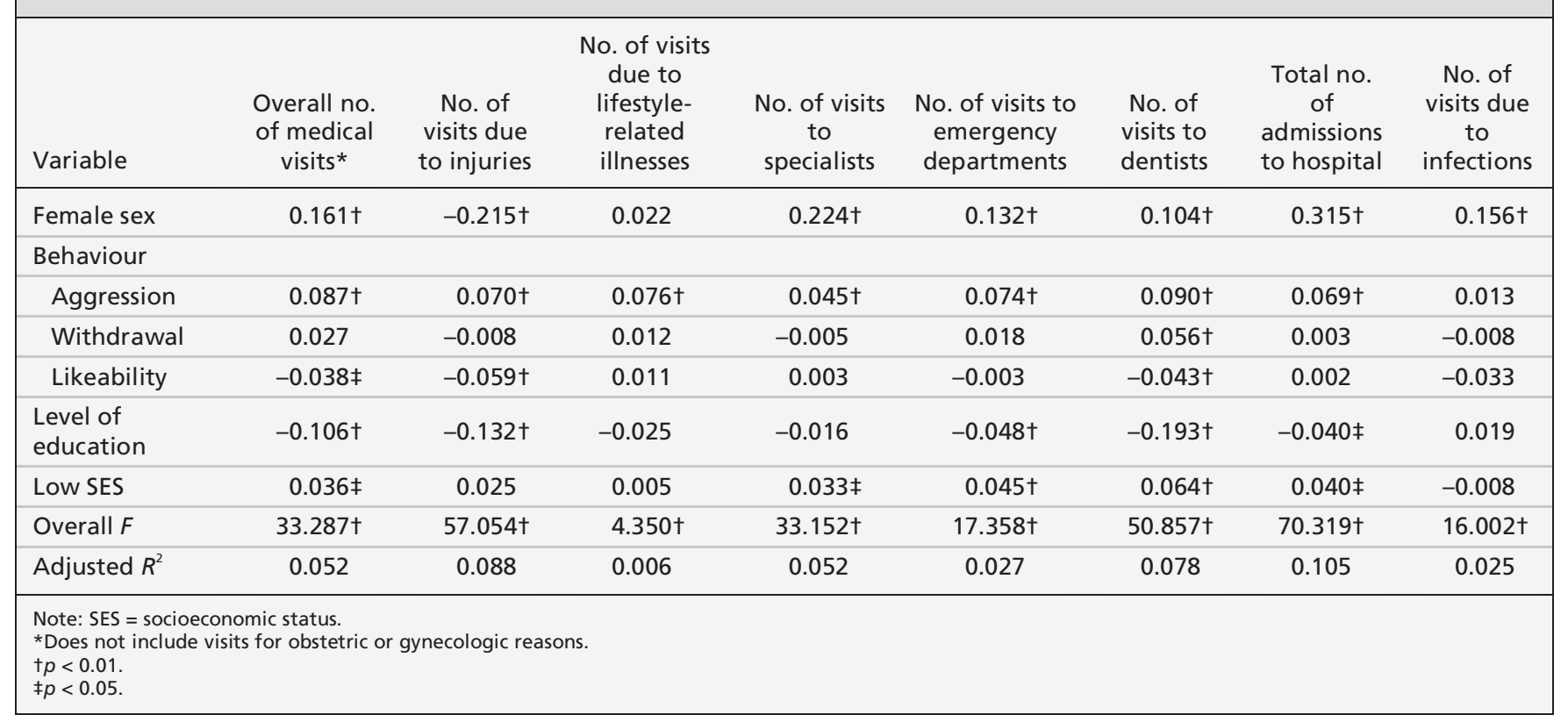




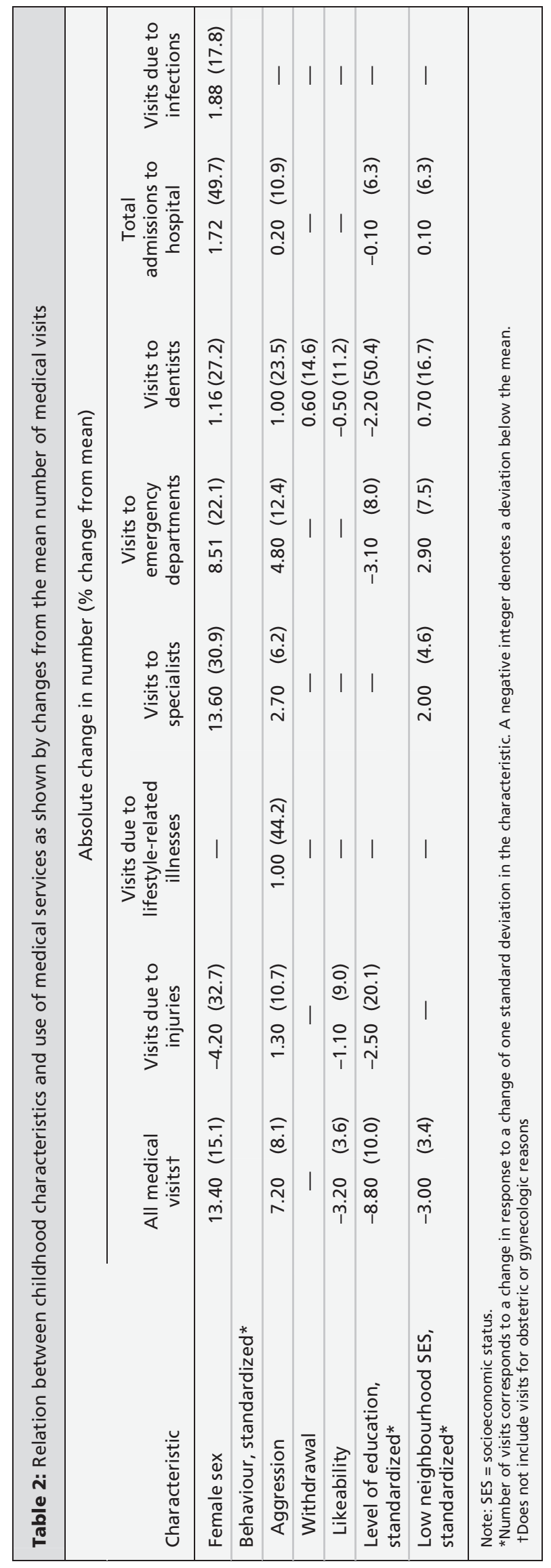

Provincial education records showed the highest level of education obtained by each participant as follows: did not complete high school, completed high school, diploma from a junior college and degree from a university.

Box 1 shows the times at which our data were collected.

\section{Statistical analysis}

We used hierarchical multiple regression, because it allowed us to examine the contribution of a particular variable while controlling for the effects of other independent variables. With the exception of gynecologic and obstetric visits, data for men and women were analyzed together.

\section{Results}

No significant interactions between sex and other predictors were seen in preliminary analyses.

We analyzed the relation between behaviour during childhood (collected 1976-1978) and physical health during adulthood (from 19922006), as well as the increase or decrease in number of medical visits, in terms of both standard deviation from the mean and percent increase or decrease from the mean, for each independent variable. Childhood aggression was positively related to overall use of health services, number of medical visits due to injuries and lifestyle-related illnesses, number of visits to specialists, emergency departments and government-funded dentists, and total number of admissions to hospital (Tables 1 and 2).

During the 15 years we included in our analyses, an increase of one standard deviation from the mean in childhood aggression corresponded to 7.2 more medical visits (an increase of $8.1 \%$ above the mean number of visits), 1.3 more visits due to injuries (10.7\% increase), 1.0 more visit for lifestyle-related illnesses (44.2\% increase), 2.7 more visits to specialists $(6.2 \%$ increase), 4.8 more visits to emergency departments (12.4\% increase), 1.0 more visit to dentists (23.5\% increase) and 0.2 more admissions to hospital (10.9\% increase) (Table 2). Conversely, childhood aggression was not related to number of medical visits due to infections.

We saw a positive relation between social withdrawal during childhood and number of dental visits ( $14.6 \%$ increase), but we saw negative relations between peer-rated likeability during childhood and overall use of medical services (3.6\% decrease), number of medical visits due to injuries (9.0\% decrease) and number of dental visits ( $11.2 \%$ decrease).

A main effect of sex was seen for overall use of medical services, number of medical visits to 
specialists, emergency departments and dentists, total number of admissions to hospital, and medical visits due to infections (Table 2). These findings suggest that women are more likely than men to seek medical attention. This effect was reversed for medical visits due to injuries, where men were more likely than women to seek medical attention (Table 2).

We saw no overall effect on use of gynecologic services when the period from 1992 to 2006 was considered (Table 3). However, previous research from this project and others suggest increases in the use of gynecologic services and higher risk of gynecologic and obstetric problems among women who show aggressive behaviour, particularly among teens and young adults. ${ }^{9}$ Therefore, we looked at the use of gynecologic services among women aged 18 to 23 years, and again when the same women were older (aged 29-34 yr). Childhood aggression was positively predictive of use of gynecologic services for the younger women, but the effect was no longer present when the same women were older (Table 3). Among younger women, an increase of one standard deviation in aggression corresponded to 0.5 more obstetric and gynecologic visits over a five-year period (ages $18-21 \mathrm{yr}$ ), representing a $5.87 \%$ increase over the mean (data not shown). Childhood likeability had a negative predictive relationship to gynecological service usage in late adolescence and early adulthood, but no relationship among the same women later in life.

Level of education showed consistently negative relations with overall use of medical services ( $10.0 \%$ decrease), number of medical visits due to injuries (20.1\% decrease), visits to emergency departments $(8.0 \%$ decrease), number of dental visits (50.4\% decrease) and total number of admissions to hospital (6.3\% decrease) (Table 2).

Living in a poorer neighbourhood during early adulthood decreased overall use of medical services by $3.4 \%$, but increased the number of visits to specialists ( $4.6 \%$ increase), emergency departments (7.5\% increase) and dentists (16.7\% increase), and total number of admissions to hospital (6.3\% increase) (Table 2). In addition, living in a poorer neighbourhood was negatively related to gynecologic visits among women in adolescence and early adulthood, but this effect was not seen among women as they grew older (Table 3).

Level of education showed significant effects during both early and later adulthood among the women in our sample. Among younger women, level of education showed a robust negative predictive effect, suggesting that young women with higher levels of education sought gynecologic services less frequently than women with less education. An increase of one standard deviation in level of education corresponded to 2.0 fewer obstetric and gynecologic visits $(25.8 \%$ decrease from the mean number of visits) (data not shown). However, among older women, this relationship was reversed; education was positively predictive of gynecologic visits, suggesting that women with higher levels of education saw their gynecologists more often than women with less education.

\section{Interpretation}

Childhood aggression directly and positively predicted overall use of health services in adulthood for the participants of this study, as well as the number of visits they made to specialists, emergency departments and dentists, the number of times they were admitted to hospital, and the number of medical visits they made due to lifestylerelated illnesses and injuries. These associations were seen even when controlling for the effects of sex, education and neighbourhood poverty.

Among young women, childhood aggression increased the use of gynecologic services. This result is consistent with previous research suggesting that agression is related to high-risk sexual behaviour early in life. ${ }^{9,10}$

Our results suggest that childhood aggression has lasting effects on physical health and can have an impact on the level of use of medical services over many years. Among the participants of our study, childhood aggression did not indiscriminately increase the number of visits to doctors, nor was it associated with an increase in

Table 3: Standardized regression coefficients showing the relation between childhood characteristics among women in two age groups and visits for gynecologic and obstetric reasons

\begin{tabular}{|lcc|}
\hline & \multicolumn{2}{c|}{ No. of visits* } \\
\cline { 2 - 3 } Characteristic & $18-23 \mathrm{yr}$ & $29-34 \mathrm{yr}$ \\
\hline Behaviour & & \\
\hline Aggression & $0.05 \dagger$ & 0.01 \\
\hline Withdrawal & 0.02 & 0.00 \\
\hline Likeability & $-0.06 \ddagger$ & 0.02 \\
\hline $\begin{array}{l}\text { Level of education } \\
\text { Low SES }\end{array}$ & $-0.22 \ddagger$ & $0.12 \ddagger$ \\
\hline $\begin{array}{l}\text { Overall } F \\
\text { Adjusted } R^{2}\end{array}$ & $-0.06 \ddagger$ & -0.02 \\
\hline $\begin{array}{l}\text { Note: SES }=\text { socioeconomic status. } \\
* \text { Number of visits corresponds to a change in response to a change of one standard } \\
\text { deviation in the characteristic. A negative integer denotes a deviation below the mean. } \\
+p<0.05 \\
\neq p<0.01\end{array}$ & $5.58 \ddagger$ \\
\hline
\end{tabular}


infections. This finding suggests that childhood aggression puts people at risk for specific types of health problems during adulthood that cause them to use medical services more frequently. Our study begins to explore how the use of specific medical services are associated with distinct behaviours.

Among our participants, childhood likeability negatively predicted overall use of medical services, number of medical visits due to injuries and number of government-funded dental visits. The direction of these effects is consistent with research suggesting that adults with larger social networks seem to have better health outcomes than those who are less socially connected. ${ }^{16,17}$ These data could also suggest that the children who were judged as more likeable by their peers were less likely to engage in risk-taking or impulsive behaviours that could lead to injuries, and more likely to take better care of their overall health. Good social relationships may also offer support and protect against the negative effects of stress, which could aid in the prevention of illness.

Childhood social withdrawal positively predicted the use of government-funded dental services. Though the mechanism underlying this relationship is ambiguous, it could be related to socioeconomic status. Alternatively, shyness and reluctance to seek regular dental care might result in a higher number of emergency dental procedures.

Level of education negatively predicted our participants' overall use of medical services, visits to emergency departments, admissions to hospital, government-funded dental visits and medical visits due to injuries. These results suggest that people with a higher level of education were less likely than those with less education to need or seek medical attention for these types of problems.

There are several possible explanations for the links seen between education and health. Leaving school prematurely may result in less education about health, which could lead to poor decision-making based on a lack of knowledge. In addition, the jobs available to people who have not finished high school often involve manual labour, which could carry an increased risk of injury. Furthermore, the stress of earning a low income associated with a lower level of education could lead to stress-related illnesses. Finally, deficits in executive functioning and impulse control may underlie both leaving school prematurely and poor adult health by exposing a person to a greater number of highrisk situations in which injury is possible and medical help is necessary.

The level of poverty of the neighbourhood in which participants lived and attended school during their late adolescence and early adulthood predicted the number of visits to specialists, emergency departments and dentists. This variable can be considered as a proxy for socioeconomic status; thus, these results are consistent with previous reports suggesting problems with primary health care among people with low incomes. For example, rates of admissions to hospital and visits to emergency departments have been shown to have a strong sociodemographic component $;{ }^{18}$ this association has been seen in Canada, where health care is universally accessible. ${ }^{19}$

Some of the variance in adult health and use of health services could be predicted from the childhood behaviour shown by the participants of this study. This finding is particularly important for primary prevention campaigns aimed at improving public health and decreasing the use of medical services and their associated costs to society. Even modest increases in the use of services in adulthood that can be predicted from childhood variables could amount to huge costs to society when multiplied over millions of people. Knowing how to target those children and adolescents most at risk for poor health later in life could help prevent certain negative health outcomes later in life, as well as potentially decrease health care costs for the Canadian public. Such a strategy requires further study.

\section{Strengths and limitations}

Longitudinal designs use a prospective approach rather than rely on retrospective data, which is subject to recall bias. Causal inferences can be drawn more credibly in prospective longitudinal studies because of the establishment of time sequencing in the prediction of outcomes. However, in most cases, conclusions remain speculative as a function of the method, which is correlational. The replication of results across studies, using different samples, is a necessary step in confirming the generality of longitudinal results. ${ }^{20}$

The effect sizes we saw were modest. However, considering that the childhood variables were collected almost 30 years before the medical data were retrieved, our results offer some insight into the long-term outcomes of childhood aggression and suggest possible avenues for preventive intervention.

\section{Conclusion}

Our results confirm that there are specific behavioural characteristics, identifiable in childhood, that can have enduring consequences to physical health and can predict increased use of health 
services in adulthood. Childhood aggression should be considered a health risk when designing interventions to improve public health, particularly those targeting children and families. To help people at risk, we should address multiple sources of risk. As such, a developmental approach to the prevention and treatment of these problems should be taken. Whether risk factors for poor health in later life can be addressed most effectively in adolescence and early adulthood or whether they should be the focus of earlier preventive interventions in childhood is an issue that needs to be addressed both empirically and from a social policy perspective. Adopting a life-long approach to teaching children, teenagers and young adults appropriate self-care and age-appropriate ways of managing their stress and impulses may effectively prevent poor health outcomes later in life.

We measured our participants' use of medical services during their early to middle adulthoods. How their health may fare later in life, when lifestyle-related illnesses such as high blood pressure, high cholesterol and coronary artery disease develop remains to be seen. Future research involving this population will help elucidate the lifelong processes of aggression, stressful life circumstances, cognitive and coping strategies, physical health consequences and use of medical services.

Our results add to the literature that suggests the people at greatest risk of poor health in adulthood, as characterized by multiple negative predictors of long-term outcome, might be identified in early childhood. Addressing problematic childhood behaviour and teaching appropriate ways of interacting, self-care and coping strategies to vulnerable children will probably require early preventive intervention to mitigate longterm risks to health.

\section{References}

1. Cairns RB, Cairns BD, Neckerman HJ. Early school dropout: configurations and determinants. Child Dev 1989;60:1436-52.

2. Bardone AM, Moffitt TE, Caspi A, et al. Adult physical health outcomes of adolescent girls with conduct disorder, depression, and anxiety. J Am Acad Child Adolesc Psychiatry 1998;37:594-601.

3. Capaldi DM, Stoolmiller M. Co-occurrence of conduct problems and depressive symptoms in early adolescent boys: III. Prediction to young-adult adjustment. Dev Psychopathol 1999;11: 59-84.

4. Bardone AM, Moffitt TE, Caspi A, et al. Adult mental health and social outcomes of adolescent girls with depression and conduct disorder. Dev Psychopathol 1996;8:811-29.

5. Odgers CL, Caspi A, Broadbent JM, et al. Prediction of differential adult health burden by conduct problem subtype. Arch Gen Psychiatry 2007;64:476-84.

6. Caspi A, Begg D, Dickson N, et al. Personality differences predict health-risk behaviours in young adulthood: evidence from a longitudinal study. J Pers Soc Psychol 1997;73:1052-63.

7. Deffenbacher JL, Deffenbacher DM, Lynch RS, et al. Anger, aggression and risky behaviour: a comparison of high and low anger drivers. Behav Res Ther 2003;41:701-18.

8. Temcheff CE, Serbin LA, Martin-Storey A. Predicting adult physical health outcomes from childhood aggression, social withdrawal and likeability: a 30-year prospective, longitudinal study. Int J Behav Med 2011 Mar; 18:5-12.

9. Serbin LA, Peters PL, McAffer VJ, Schwartzman AE. Childhood aggression and withdrawal as predictors of adolescent pregnancy, early parenthood, and environmental risk to the next generation. Can J Behav Sci 1991;23:318-31.

10. Serbin LA, Cooperman JM, Peters PL, et al. Intergenerational transfer of psychosocial risk in women with childhood histories of aggression, withdrawal, or aggression and withdrawal. Dev Psychol 1998;34:1246-62.

11. Bardone AM, Moffitt TE, Caspi A, et al. Adult physical health outcomes of adolescent girls with conduct disorder, depression, and anxiety. J Am Acad Child Adolesc Psychiatry 1998;37:594-601.

12. Underwood MK, Kupersmidt JB, Coie JD. Childhood peer sociometric status and aggression as predictors of adolescent childbearing. J Res Adolesc 1996;6:201-23.

13. Xie H, Cairns BD, Cairns RB. Predicting teen motherhood and teen fatherhood: individual characteristics and peer affiliations. Soc Dev 2001; 10:488-511.

14. Pekarik EG, Prinz RJ, Liebert DE, et al. The Pupil Evaluation Inventory: a sociometric technique for assessing children's social behaviour. J Abnorm Child Psychol 1976;4:83-97.

15. Schwartzman AE, Ledingham J, Serbin LA. Identification of children at risk for adult schizophrenia: a longitudinal study. Int J App Psychol 1985;34:363-79.

16. Uchino BN. Social support and health: a review of physiological processes potentially underlying links to disease outcomes. $J$ Behav Med 2006;29:377-87.

17. Berkman LF, Glass T. Social integration, social networks, social support \& health. In: Berkman LF, Kawachi I, editors. Social epidemiology. New York (NY): Oxford University Press; 2000.

18. Billings J, Zeitel L, Lukomnik J, et al. Impact of socioeconomic status on hospital use in New York City. Health Aff (Millwood) 1993;12:162-73.

19. Roos LL, Walld R, Uhanova J, et al. Physician visits, hospitalizations, and socioeconomic status: ambulatory care sensitive conditions in a Canadian setting. Health Serv Res 2005;40:1167-85.

20. Serbin L, Karp J. Intergenerational studies of parenting and the transfer of risk from parent to child. Curr Dir Psychol Sci 2003; 12:138-42.

Affiliations: From the Département de psychoéducation (Temcheff), Université de Sherbrooke, Sherbrooke, Que.; The Department of Psychology, Concordia University and Center for Research in Human Development (Serbin, MartinStorey, Stack, Schwartzman) Montréal, Que.; the Department of Psychology (Hastings), University of California, Davis, Calif.; and the School of Psychology (Ledingham), University of Ottawa, Ottawa, Ont.

Contributors: Caroline Temcheff, Lisa Serbin and Alex Schwartzman conceived the original idea for the article. Caroline Temcheff analyzed the data, and wrote and revised the manuscript. Lisa Serbin obtained funding for the study and revised the manuscript. Alexa Martin-Storey assisted with writing and revising the manuscript. Dale Stack and Paul Hastings obtained funding for the study and reviewed the manuscript. Jane Ledingham and Alex Schwartzman collected the original data and reviewed the manuscript.

Funding: This research was supported by a grant from the Canadian Institutes of Health Research (grant no. MOP82876). This research was made possible through the support of the Commission d'accès à l'information, who granted the approval to gain access to all relevant information (reference no. 070871).

Acknowledgements: The authors thank Claude Senneville and the Concordia project team for their assistance in data collection and analysis; the participants of the study; and the Régie de l'assurance maladie du Québec, the Ministère de la Santé et des services sociaux and the Ministère de l'éducation, des loisirs et du sport, who provided the data necessary for the study. 